\title{
Determination of electron and hole effective masses in thermal oxide utilizing an n-channel silicon MOSFET
}

\author{
Ravi Kumar Chanana \\ Department of Electrical and Electronics Engineering, Galgotia's College of Engineering and Technology, \\ Affiliated to Uttar Pradesh Technical University, Lucknow, India. \\ 1, Institutional Area, Knowledge Park-II, Greater Noida - 201306, India.
}

\begin{abstract}
The gate tunnelling electron current and the substrate hole current obtained from carrier separation in an n-channel Si MOSFET are used to determine the parabolic electron and hole effective masses in the thermal oxide. The oxide voltages for electron and hole conduction in the n-MOSFET in inversion are formulated, and the carrier effective masses of $0.42 \mathrm{~m}$ for electron and $0.58 \mathrm{~m}$ for hole for a free Fermi gas model of carriers at the emitting electrode are determined using the Fowler-Nordheim tunnelling characteristics. These carrier masses are related to the band offsets in $\mathrm{SiO}_{2} / \mathrm{Si}\langle 100\rangle \mathrm{MOS}$ devices accurately through the MOSFET model for the first time. The carrier effective masses are predicted to be the same for all thickness of oxide. The technique can be extended to other insulating materials as well. Also, the 1/E model of the anode hole injection over the hole barrier of $4.6 \mathrm{eV}$ completely explains the oxide breakdown in thin oxides of 5 to $10 \mathrm{~nm}$ at high electric fields, and having a slope constant of $516 \mathrm{MV} / \mathrm{cm}$.
\end{abstract}

Keywords: effective mass, $F N$-tunelling, band offsets, metal-insulator-semiconductor

\section{Introduction}

The knowledge of electron and hole effective masses and conduction and valence band offset values in a metal-oxide-semiconductor (MOS) device can facilitate simulation of Fowler-Nordheim (FN) tunnelling currents through a MOS device at high fields. The FN onset field and the dielectric breakdown field can also be determined. The FN onset field can be obtained for a minimum displacement current density of $10^{-8}-10^{-9} \mathrm{~A} / \mathrm{cm}^{2}$, and the dielectric breakdown field can be obtained for a current density of $10^{-4} \mathrm{~A} / \mathrm{cm}^{2}$, with the above knowledge. The onset field is the upper limit to which the oxide can work as a good insulator without injection and trapping of carriers in it. The trapping of carriers causes degradation of the oxide and reduces its reliability. The above knowledge of carrier effective masses and band offsets can also be utilized to determine oxide thickness to within $0.3 \mathrm{nmof}$ other physical techniques of thickness measurement [1]. In view of the above, the determination of the electron and hole effective masses and band offset values in a MOS device is of utmost importance. These tunnelling parameters can be determined from the FN tunnelling equation. The equation models the current-voltage characteristics across a MOS device at high fields [2]. The electron effective mass in the thermal oxide as insulator has been determined earlier to be $0.42 \mathrm{~m}$, where $\mathrm{m}$ is the free electron mass based on the free Fermi gas model for the carriers at the emitting electrode of a MOS device in accumulation $[2,3]$. In the present study, the carrier effective masses in the thermal oxide are determined utilizing the gate tunnelling electron current and the substrate hole current versus the oxide voltage characteristics of a MOSFET. These currents are obtained from carrier separation in an n-channel silicon MOSFET device in inversion [2], with the oxide bandgap value taken as $8.9 \mathrm{eV}[4,5]$. The carrier mass values obtained are $0.42 \mathrm{~m}$ for the electron effective mass and $0.58 \mathrm{~m}$ for the hole effective mass for a free Fermi gas model of carriers at the emitting electrode. However, if quantum confinement of carriers is considered at the emitting electrode, where the conduction or valence band edge forms a triangular potential well when biased, then these values have to be corrected. Nearly $70 \%$ population of carriers is calculated to be residing at the ground state subband energy level in the potential well [6], which reduces the electron barrier to oxide conduction band by $0.2 \mathrm{eV}$ [7], and the hole barrier to oxide valence band by $0.16 \mathrm{eV}$ [8] in $\mathrm{Si}<100>\mathrm{MOS}$ devices. The corrected electron and hole barrier heights due to quantum confinement results in the electron and hole effective masses in the thermal oxide to $0.51 \mathrm{~m}$ and $0.65 \mathrm{~m}$ respectively. It needs to be mentioned here that quantum confinement which causes bandgap broadening at the semiconductor-oxide interface and affects the carrier effective mass calculations, is valid for extremely thin channels in the MOSFETs when the peak of the electron concentration lie several angstroms away from the $\mathrm{Si} / \mathrm{SiO}_{2}$ interface and thus reduces the saturation current. To the author's knowledge to date, quantum correction models, such as the triangular potential well model used by Weinberg [7] has not been able to reproduce the reduced saturation current characteristics in the MOSFETs having thin channels. 
Determination of electron and hole effective masses in thermal oxide utilizing an $n$-channel...

I. Theory:

FN electron and hole tunnelling has been observed in $\mathrm{Si}$ and $\mathrm{SiC}$ MOS devices and in organic light emitting diodes [9-12]. The FN equation models the current-voltage characteristics across a MOS device at high fields. It is given by the classical equation $[7,10]$ :

$\frac{J}{E^{2}}=A \exp \left(\frac{-B}{E}\right) ;(1)$

where $\mathrm{J}$ is the current density across the MOS device in $\mathrm{A} / \mathrm{cm}^{2}, \mathrm{E}$ is the oxide electric field in $\mathrm{V} / \mathrm{cm}$, and the preexponent $\mathrm{A}$ and the slope constant $\mathrm{B}$ are given by:

$$
\begin{aligned}
A & =\frac{e^{3} m}{16 \pi^{2} \hbar m_{o x} \varphi_{0}} \\
A & =1.54 \times 10^{-6} \frac{m}{m_{o x}} \frac{1}{\varphi_{0}}\left(A / V^{2}\right), \\
B & =\frac{4}{3} \frac{\left(2 m_{o x}\right)^{1 / 2}}{e \hbar} \varphi_{0}^{3 / 2} \\
B & =6.83 \times 10^{7}\left(\frac{m_{o x}}{m}\right)^{1 / 2} \varphi_{0}^{3 / 2}(\mathrm{~V} / \mathrm{cm})(3)
\end{aligned}
$$

In $A$ and $B$ constants, $\mathrm{e}$ is the electronic charge, $\mathrm{m}$ is the free electron mass, $\mathrm{m}_{\mathrm{ox}}$ is the electron or hole mass in the oxide, $2 \pi \hbar$ is Planck's constant and $\varphi_{0}$ is the electron or hole barrier height expressed in electron volts. A plot of $\ln \left(\mathrm{J}^{\mathrm{E}} \mathrm{E}^{2}\right)$ versus $1 / \mathrm{E}$, called an FN plot, gives the value of the slope constant $\mathrm{B}$, from which $\left(m_{o x} / m\right)^{1 / 2} \varphi_{0}^{3 / 2}$ product can be obtained. Then, with a known effective mass, $\varphi_{0}$ can be calculated, and with a known $\varphi_{0}$, the effective mass in the oxide can be calculated. The slope constant B is very sensitive to the oxide field as it is in the exponential and therefore precise determination of the oxide field is absolutely critical in the evaluation of the tunnelling parameters. The $\ln \left(\mathrm{J} / \mathrm{E}^{2}\right)$ term is relatively much less sensitive to the oxide field as it is in the natural logarithm.

\section{A. Formulation of the oxide voltage in a n-channel-MOSFET:}

In an ideal MOS diode, if the applied voltage across the diode is $\mathrm{V}$, then $-Q_{d} / C_{i}$ is the voltage across the oxide [13]. For an $n$-channel MOSFET $\mathrm{Q}_{\mathrm{d}}$ is negative, giving a positive voltage across the oxide insulator. The threshold voltage for strong inversion, $\mathrm{V}_{\mathrm{T}}$ for a practical MOS device is expressed as:

$V_{T}=\varphi_{m s}-\left(\frac{Q_{i}}{C_{i}}\right)-\left(\frac{Q_{d}}{C_{i}}\right)+\psi_{s}-\psi_{\text {poly }}$;

where, $\varphi_{m s}$ is the metal-semiconductor work function difference, $\mathrm{Q}_{\mathrm{i}}$ is the oxide insulator charge density, $\mathrm{C}_{\mathrm{i}}$ is the oxide insulator capacitance per unit area, $\mathrm{Q}_{\mathrm{d}}$ is the depletion charge density in the semiconductor, and $\psi_{s}$ is twice the bulk potential in the semiconductor at which strong inversion occurs for the MOS device and $\psi_{\text {poly }}$ is the polysilicon depletion potential. For an applied voltage $\mathrm{V}_{\mathrm{T}}$ across the MOS device, ideally $-\mathrm{Q}_{\mathrm{d}} / \mathrm{C}_{\mathrm{i}}$ will fall across the oxide. So, the voltage across the oxide $-\mathrm{Q}_{\mathrm{d}} / \mathrm{C}_{\mathrm{i}}$ can be expressed as:

$V_{o x}=V_{T}-V_{f b}-\psi_{s}+\psi_{\text {poly }}$;

where $V_{f b}=\varphi_{m s}-\left(Q_{i} / C_{i}\right)$ is the flatband voltage. Next, if the applied voltage is $V-V_{s b}$, then the expression for the oxide voltage becomes:

$V_{o x}=V-V_{s b}-V_{f b}-\psi_{s}+\psi_{\text {poly }}$.

Here, $V_{s b}$ is the reverse bias applied to the substrate of a MOSFET. It is used to control the threshold voltage in a MOSFET. This is the same equation as developed by Depas and co-researchers [14], except that $\mathrm{V}_{\mathrm{sb}}$ is also part of the equation for a MOSFET. In terms of the threshold voltage $\mathrm{V}_{\mathrm{T}}$, the equation can be written as:

$V_{o x}=V-V_{s b}-V_{T}-\frac{Q_{d}}{C_{i}}$;

For an n-channel MOSFET in inversion, $\mathrm{V}_{\mathrm{sb}}$ is negative, $\mathrm{V}_{\mathrm{T}}$ is positive, and $\mathrm{Q}_{\mathrm{d}}$ is negative. Also, $\mathrm{Q}_{\mathrm{d}}$ gets modified due to the application of $\mathrm{V}_{\mathrm{sb}}$. Therefore, utilizing the absolute voltages for an $\mathrm{n}$-channel MOSFET, the expression for the oxide voltage for electron tunnelling across the ideal oxide that does not contain any positive charges, can be written as:

$V_{o x}=|V|+\left|V_{s b}\right|-\left|V_{T}\right|+\left|\frac{Q_{d}}{C_{i}}\right|$.

The electron tunnelling across the oxide is represented by the gate current in the n-channel MOSFET [9]. The substrate hole current in the n-channel MOSFET [9] is believed to be due to the back injection of hot holes from the polysilicon anode having the hole barrier of $4.6 \mathrm{eV}$ from the valence band of the polysilicon anode to the oxide valence band [15]. Once the hot holes come into the valence band over the barrier [15], the hole current follows the exp(-B/E) dependence of the FN tunnelling equation (1) through the oxide. An nchannel MOSFET having substrate hole conduction is identical to a p-channel MOSFET having hole injection followed by conduction with the $\psi_{s}$ and $\psi_{\text {poly }}$ of p-MOSFET changed as $\psi_{\text {poly }}$ and $\psi_{s}$ of n-MOSFET. This can be observed from Fig.1(a) and (b). Fig. 1(a) shows an n-MOSFET and Fig.1 (b) shows a p-MOSFET. It can be observed that $\psi_{s}$ and $\psi_{\text {poly }}$ of the p-MOSFET can be changed to $\psi_{\text {poly }}$ and $\psi_{s}$ for the n-MOSFET respectively making the n-MOSFET with hole conduction similar to p-MOSFET with hole conduction. 
Therefore, the oxide voltage across the n-MOSFET for substrate hole current conduction can be obtained from the oxide voltage across the p-MOSFET. In a p-MOSFET, the threshold voltage equation (4) given above has negative $\mathrm{V}_{\mathrm{T}}$, negative $\psi_{\mathrm{s}}$ and positive $\mathrm{Q}_{\mathrm{d}}$, and

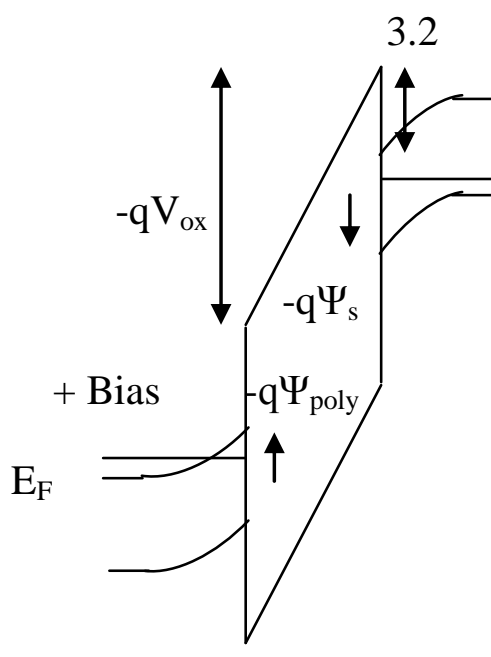

(a)
- Bias
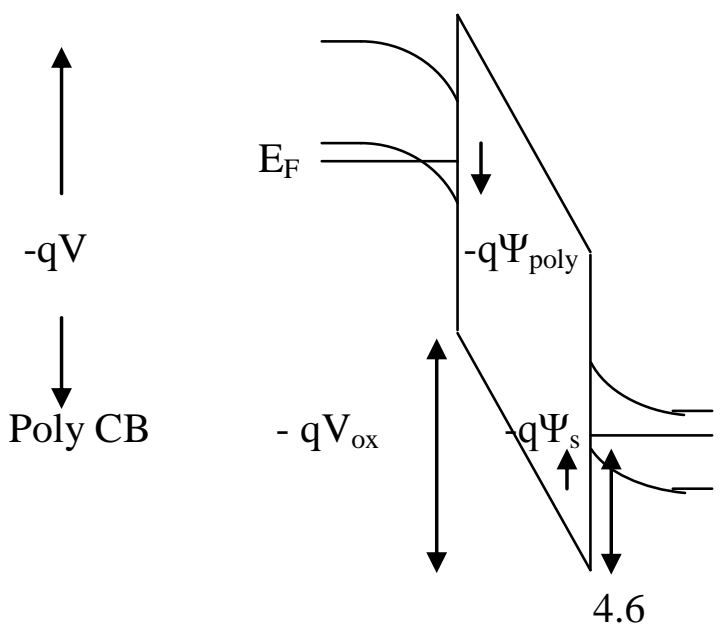

Poly VB

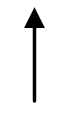

$-\mathrm{qV}$

$$
\mathrm{n}^{+} \text {Poly } \quad \mathrm{SiO}_{2} \quad \mathrm{p}-\mathrm{Si} \quad \mathrm{p}^{+} \text {Poly } \quad \mathrm{SiO}_{2} \quad \mathrm{n}-\mathrm{Si}
$$

Fig. 1. Energy-band diagram for a (a) n-MOSFET with $\mathrm{n}^{+}$polysilicon gate and for a

(b) $\mathrm{p}$-MOSFET with $\mathrm{p}^{+}$polysilicon gate. Both the devices are biased in inversion.

assuming that the magnitude of $\mathrm{V}_{\mathrm{T}}$ for $\mathrm{n}$ - and p-MOSFETs is the same. Therefore, for positive oxide charges, the threshold voltage equation (4) for the p-MOSFET can be written as:

$-\left|V_{T}\right|=-\left|V_{f b}\right|-\left|\psi_{s}\right|-\left|\frac{Q_{d}}{C_{i}}\right|+\left|\psi_{\text {poly }}\right|$;

Interchanging $\psi_{s}$ and $\psi_{\text {poly }}$ and multiplying throughout by -1 , gives:

$\left|V_{T}\right|=\left|V_{f b}\right|-\left|\psi_{s}\right|+\left|\frac{Q_{d}}{C_{i}}\right|+\left|\psi_{\text {poly }}\right|$

Here, for $\mathrm{V}_{\mathrm{T}}$ as the applied voltage, $\mathrm{Q}_{\mathrm{d}} / \mathrm{C}_{\mathrm{i}}$ is the ideal drop across the oxide. This drop in voltage for an applied voltage $\mathrm{V}_{\mathrm{T}}$ can be written as $\mathrm{V}_{\mathrm{ox}}$. Therefore, for $|V|+\left|V_{s b}\right|$ as the applied voltage, the oxide voltage drop across the n-MOSFET with hole conduction can be written as:

$V_{o x}=|V|+\left|V_{s b}\right|-\left|V_{f b}\right|+\left|\psi_{s}\right|-\left|\psi_{\text {poly }}\right|$;

For the sake of simplified intermediate steps in formulation of the corrected oxide voltage, the $\mathrm{V}_{\mathrm{fb}}$ for $\mathrm{n}$ - and p-MOSFET is assumed to be the same. Now, from equation (4) for $\mathrm{V}_{T}$ across an n-MOSFET, where $\mathrm{Q}_{\mathrm{d}}$ is negative, $\psi_{s}$ is positive, and with the oxide having positive charges;

$-\left|V_{f b}\right|+\left|\psi_{s}\right|-\left|\psi_{\text {poly }}\right|=\left|V_{T}\right|-\left|\frac{Q_{d}}{C_{i}}\right|$;

Substituting equation (12) in equation (11) gives the equation for $\mathrm{V}_{\mathrm{ox}}$ in n-MOSFET having hole conduction as: $V_{o x}=|V|+\left|V_{s b}\right|+\left|V_{T}\right|-\left|\frac{Q_{d}}{C_{i}}\right|$.

Thus, for an n-channel MOSFET device, equation (8) represents the corrected oxide voltage for electron conduction through the oxide, and equation (13) represents corrected oxide voltage for substrate hole conduction through the oxide. The addition of $\mathrm{V}_{\mathrm{T}}$ for hole conduction in equation (13) suggests that $\varphi_{m s}$ for electron conduction becomes $\varphi_{s m}$ for hole conduction. The oxide voltage for electron conduction is therefore different from the oxide voltage for hole conduction. This point is also discussed in the authors's earlier work [2]. It is shown that the oxide voltage has to be corrected by the flatband voltage for MOS devices in accumulation. In 
Determination of electron and hole effective masses in thermal oxide utilizing an $n$-channel...

the present study utilizing an n-channel MOSFET, the oxide voltages are modified by the threshold voltage.

\section{Sample Calculations and Results:}

A sample calculation for the electron and hole effective masses is performed here, utilizing the currentvoltage data on an $\mathrm{n}^{+}$-polysilicon gated $\mathrm{n}$-channel Si MOSFET. Two points of current and voltage are taken in the FN regime for the gate current representing electron tunnelling current and the substrate hole current from fig. 2 of reference [9], and are presented in Table I below.

Table I. Current-voltage data in the FN region for gate electron current and substrate hole current of the MOSFET.

\begin{tabular}{|c|c|c|c|c|c|}
\hline Current Type & (V) ${ }^{\left|V_{1}\right|+\left|V_{s b}\right|}$ & $\begin{array}{l}\mathrm{I}_{1} \\
(\mathrm{~A})\end{array}$ & (V) $\left|V_{2}\right|+\left|V_{s b}\right|$ & $\begin{array}{l}\mathrm{I}_{2} \\
(\mathrm{~A})\end{array}$ & $\begin{array}{ll}\begin{array}{l}\text { Uncorrected } \\
(\mathrm{MV} / \mathrm{cm})\end{array} & \mathrm{B} \\
\end{array}$ \\
\hline $\begin{array}{l}\text { Gate Electron } \\
\text { Current Data }\end{array}$ & $(8.0+1)$ & $10^{-8}$ & $(9.5+1)$ & $10^{-6}$ & 330 \\
\hline $\begin{array}{ll}\text { Substrate } & \text { Hole } \\
\text { Current Data } & \\
\end{array}$ & $(9.5+1)$ & $10^{-9}$ & $(11.0+1)$ & $10^{-7}$ & 434 \\
\hline
\end{tabular}

For the calculation of corrected oxide voltages, the threshold voltage $\mathrm{V}_{\mathrm{T}}$ is taken as $1.25 \mathrm{~V}$. The substrate bias adjusts the threshold voltage of the MOSFET. As the magnitude of the substrate bias $\mathrm{V}_{\mathrm{sb}}$ increases from $1 \mathrm{~V}$ to $16 \mathrm{~V}, \mathrm{~V}_{\mathrm{T}}$ also increases from $1.25 \mathrm{~V}$ to $3.25 \mathrm{~V}$ as presented in fig. 43 of reference [16]. Thus, $\mathrm{V}_{\mathrm{T}}$ is $1.25 \mathrm{~V}$ for the substrate bias of $-1 \mathrm{~V}$, in the present study. The $\mathrm{V}_{\mathrm{T}}$ values presented in the fig. 43 of the above reference [16] are considered more accurate because they are calculated experimentally using the MOSFET channel conductance. The other desired parameter values are calculated and presented in Table II below.

Table II. MOSFET parameters for the calculations of corrected oxide voltages.

\begin{tabular}{|l|l|l|}
\hline $\begin{array}{l}\text { Substrate doping } \\
\mathrm{N}_{\mathrm{A}}=10^{16} / \mathrm{cm}^{3}\end{array}$ & $\begin{array}{l}\text { Intrinsic carrier conc. } \\
\mathrm{n}_{\mathrm{i}}=1.45 \times 10^{10} / \mathrm{cm}^{3}\end{array}$ & $\begin{array}{l}\text { Surface potential at strong inversion } \\
\psi_{s}=2 \psi_{B}=\frac{2 k T}{q} \ln \frac{N_{A}}{n_{i}}=0.7 \mathrm{~V}\end{array}$ \\
\hline$\varepsilon_{0}=8.854 \times 10^{-14} \mathrm{~F} / \mathrm{cm}$ & $\begin{array}{l}\text { Oxide capacitance } \\
\varepsilon_{r}=3.9 \\
\text { Oxide thickness } \\
\mathrm{d}=85 \times 10^{-8} \mathrm{~cm}\end{array}$ & $\begin{array}{l}C_{i}=\frac{\varepsilon_{0} \varepsilon_{r}}{d} \\
=4.0 \times 10^{-7} \mathrm{~F} / \mathrm{cm}^{2}\end{array}$ \\
\hline$\varepsilon_{S i}=11.9 \varepsilon_{0}$ & $\begin{array}{l}\text { Depletion charge density } \\
Q_{d}=\sqrt{2 \varepsilon_{S i} q N_{A}\left(\left|\psi_{s}\right|+\left|V_{s b}\right|\right)}\end{array}$ & $\frac{Q_{d}}{C_{i}}=0.19 \mathrm{~V}$ \\
\hline $\begin{array}{l}\mathrm{n}^{+} \text {poly Si gate and p-Si work } \\
\text { function difference } \\
\phi_{m s}=-1.08 \mathrm{~V}\end{array}$ & $\begin{array}{l}\text { Oxide fixed charge density } \\
Q_{i}=q .2 \times 10^{10} \mathrm{C} / \mathrm{cm}^{2}\end{array}$ & $\begin{array}{l}\text { Flatband voltage } \\
V_{f b}=\phi_{m s}-\frac{Q_{i}}{C_{i}}=-1.1 \mathrm{~V}\end{array}$ \\
\hline
\end{tabular}

Utilizing the values of the above parameters, the corrected oxide voltages can be calculated. The corrected slope constant B is determined from the I-V characteristics using equation (1) given above. First, $\Delta \ln \left(J / E^{2}\right) / \Delta(1 / E)$ is calculated by taking at least two points on the I-V characteristics in the FN regime at high fields given in Table I. For this, the sum of the gate voltage and substrate bias voltage is used for a particular gate current or substrate current in case of the n-channel MOSFET device. This yields the uncorrected B presented in Table I. Next, the corrected B is calculated by dividing $\Delta \ln \left(J / E^{2}\right)$ by $\Delta(1 / E)$, where $\Delta \ln \left(J / E^{2}\right)$ is the same that was used for the calculation of uncorrected $\mathrm{B}$, but the $\Delta(1 / E)$ employs the corrected oxide voltages. This is because $\Delta \ln \left(J / E^{2}\right)$ does not change with the oxide field, as it is in the natural logarithm, and the oxide voltages are corrected for the same current density $\mathbf{J}$ as for uncorrected B. The corrected oxide voltages for the gate electron current and the substrate hole current are given in Table III. These values of oxide voltages are obtained by using equation (8) and (13). The oxide voltage divided by the oxide thickness gives the corrected oxide field. Finally, from the corrected $\mathrm{B}$ value, $\mathrm{m}_{\mathrm{ox}}$ or $\varphi_{0}$ can be determined if one of them is known using equation (3). The corrected oxide voltages, the corrected slope constant B, the electron and hole barrier heights used, and the calculated effective masses $\mathrm{m}_{\mathrm{ox}}$, for electron and hole are presented in Table III below. 
Determination of electron and hole effective masses in thermal oxide utilizing an $n$-channel...

Table III. Corrected oxide voltages and tunnelling parameters for gate electron and substrate hole currents in the MOSFET.

\begin{tabular}{|l|l|l|l|l|l|}
\hline Current Type & Corrected $\mathrm{V}_{\mathrm{ox} 1}(\mathrm{~V})$ & Corrected $\mathrm{V}_{\mathrm{ox} 2}(\mathrm{~V})$ & Corrected B $(\mathrm{MV} / \mathrm{cm})$ & $\varphi_{0}(\mathrm{eV})$ & $m_{\text {ox }}(\mathrm{determined})$ \\
\hline Gate Electron Current Data & 7.94 & 9.44 & 254 & 3.2 & $0.42 \mathrm{~m}$ \\
\hline Substrate Hole Current Data & 11.56 & 13.06 & 516 & 4.6 & $0.58 \mathrm{~m}$ \\
\hline
\end{tabular}

The calculated electron effective mass in the thermal oxide for an electron barrier of $3.2 \mathrm{eV}$ from the $\mathrm{Si}$ conduction band to oxide conduction band is $0.42 \mathrm{~m}$. The hole effective mass in the oxide for an anode hole barrier of $4.6 \mathrm{eV}$ is determined to be $0.58 \mathrm{~m}$. For quantum confinement at the emitting electrode when biased, the reduced electron and hole barrier heights give electron effective mass in the oxide as $0.51 \mathrm{~m}$ and the hole effective mass as $0.65 \mathrm{~m}$. The carrier effective mass values for a free Fermi gas model for carriers at the emitting electrode are the same as that obtained in a recent study using $\mathrm{n}-$ and $\mathrm{p}-4 \mathrm{H}-\mathrm{SiC}$ MOS devices in accumulation with $\mathrm{SiO}_{2} / 4 \mathrm{H}-\mathrm{SiC}$ devices having vastly different band offsets than $\mathrm{SiO}_{2} / \mathrm{Si}<100>$ devices [2]. This reaffirms the values and also suggests that an n-channel Si MOSFET device that allows carrier separation can be used to determine the carrier effective mass values accurately.

\section{Discussion}

FN tunnelling of carriers occurs in thick oxides greater than $4 \mathrm{~nm}[1,14,17]$ in which the application of high electric field results in carrier tunnelling as a wave into the oxide conduction or valence band through the triangular barrier and then travels through the insulator as a particle with an effective mass to reach the opposite electrode. In oxides having thickness of $4 \mathrm{~nm}$ or less, the electron or hole directly tunnels across the trapezoidal barrier of the MOS device to the opposite electrode as a wave without going to the oxide conduction or valence band. Thus, the carrier propagates as a wave-particle dual in FN tunnelling, and as a wave in direct-tunnelling across the MOS device. In either case the propagation is through the oxide. Therefore, the author predicts that the carrier effective masses will remain the same for all the thicknesses of the oxide or insulating materials. A recent report has modelled the direct and FN-tunneling of electrons utilizing a thickness independent tunnel mass of $0.42 \mathrm{~m}$ [18]. Another report presents the electron effective mass in a thin tunnel oxide of $3.5 \mathrm{~nm}$ as $0.42 \mathrm{~m}[19]$, which is the same as that in thick oxides[2,3].

The origin of the substrate current in n-MOSFETs having thin oxide from 5 to $10 \mathrm{~nm}$ is under debate [9, 15, 20, 21]. Eitan and Kolodny were one of the first to observe substrate hole current in n-channel MOSFET having $8.5 \mathrm{~nm}$ oxide, and attributed the hole current to the valence electron tunnelling from the Si substrate having a barrier of $4.3 \mathrm{eV}$ from the $\mathrm{Si}$ valence band to the oxide conduction band [9]. DiMaria et al. proposed later that these holes originate as hot holes from the polysilicon anode. The energy for the hot holes are provided by the FN tunnelling electrons from the cathode, which have a threshold average energy of about $5 \mathrm{eV}$ from the bottom of the oxide conduction band. These hot holes are concluded to be back injected from the anode over the hole barrier at the $\mathrm{Si}$ anode for thin oxides of 5 to $10 \mathrm{~nm}$. In these thin oxides, the electron transport is quasiballistic, so that the maximum electron energy at the anode is independent of the oxide thickness giving a thickness independent threshold [15]. More recently, the substrate holes are experimentally shown to be generated by FN-induced photons in the polysilicon gate [20]. A report after the above, refuted this process of hole generation and concluded that the generation efficiency of photons with energy above the Si bandgap energy is $10^{-4}$ times smaller than that of the electron-hole pairs by impact ionization [21]. The above studies, leads the author to believe that the hot holes from the anode are back injected into the oxide over the barrier [15, 22]. After coming to the oxide valence band, the hole current follows the $\exp (-\mathrm{B} / \mathrm{E})$ dependence of the FN tunnelling equation (1). The calculated hole effective mass based on this dependence is exactly the same as that determined from the FN tunnelling of holes observed in p-4H-SiC MOS devices in accumulation [2]. This value of hole effective mass is $0.58 \mathrm{~m}$ for a free Fermi gas model of carriers at the emitting electrode. A value of $0.57 \mathrm{~m}$ has been used earlier as a fitting parameter in a high frequency tunnel emitter transistor model [23]. The value is also consistent with the evidence of light holes near the top of the oxide valence band [24].

The valence band offset of $4.6 \mathrm{eV}\left(\mathrm{E}_{\mathrm{g}}\right.$,oxide $-\mathrm{E}_{\mathrm{g}, \mathrm{Si}}-\mathrm{Si} \mathrm{CB}$ offset) results in the hole effective mass value of $0.58 \mathrm{~m}$ in the oxide. This valence band offset has also been reported recently in experimental studies on remote plasma grown dry $\mathrm{SiO}_{2}$ of 0.9 to $2.2 \mathrm{~nm}$, and $2 \mathrm{~nm}$ dry thermal $\mathrm{SiO}_{2}$ utilizing soft x-ray photoemission spectroscopy on $\mathrm{SiO}_{2} / \mathrm{Si}(100)$ test samples. Table I of the reference [25] presents the valence band offset for the remote plasma $\mathrm{SiO}_{2} / \mathrm{Si}(100)$ sample as $4.54 \pm 0.06 \mathrm{eV}$, and the more recent study on $2 \mathrm{~nm}$ dry thermal $\mathrm{SiO}_{2} / \mathrm{Si}(100)$ sample presents the valence band offset as $4.5 \pm 0.1 \mathrm{eV}$ [26]. The resulting hole mass of $0.58 \mathrm{~m}$ is the same as that obtained for p-4H-SiC MOS device in accumulation undergoing FN tunnelling of holes [2], where the Schottky barrier lowering does not change the tunnelling distance. This implies that Schottky barrier lowering in thin oxides of 5 to $10 \mathrm{~nm}$, in which the hot holes are injected into the oxide valence band by thermionic emission over the barrier is absent $[15,22]$. This is due to the fact that the generated hot holes due to impact ionization at the polysilicon anode of the electrons arriving from the cathode also have a thickness 
Determination of electron and hole effective masses in thermal oxide utilizing an $n$-channel...

independent energy. This eliminates any Schottky barrier lowering at the valence band which is field-dependent [22] and therefore thickness-dependent.

The oxide breakdown in thin oxide films of 5 to $10 \mathrm{~nm}$ is intimately related to the substrate current due to hot holes, which is proportional to $\exp (-\mathrm{B} / \mathrm{E})$ at high electric fields with the slope constant $\mathrm{B}$ of $516 \mathrm{MV} / \mathrm{cm}$ presented in Table III. An n-channel silicon MOSFET when biased in inversion as shown in Fig.1a, results in FN tunnelling of electrons from the cathode into the oxide due to the smaller electron barrier to oxide conduction band of $3.2 \mathrm{eV}$. They then arrive at the polysilicon anode, where they impact ionize and create hot holes. The hot holes inject over the hole barrier of $4.6 \mathrm{eV}$ at the anode [15] and cause the substrate hole current through the oxide. Some holes are trapped in the oxide causing increase in the field at the cathode [2]. This results in larger electron current injection from the cathode into the oxide by FN tunnelling followed by increased substrate current and hole trapping in the oxide. This positive feedback results in the oxide breakdown. The time-to-breakdown is therefore proportional to $\exp (-\mathrm{B} / \mathrm{E})$ with the slope constant $\mathrm{B}$ of 516 $\mathrm{MV} / \mathrm{cm}$ instead of $350 \mathrm{MV} / \mathrm{cm}$ reported earlier [27]. This 1/E model of the anode hole injection [27] completely explains the oxide breakdown in thin oxide films of 5 to $10 \mathrm{~nm}$ at high electric fields.

Several MOS device configurations can be used to determine electron and hole effective masses or band offsets in thermal oxide and in other viable high-K dielectrics [26, 28, 29]. Six of them are listed below:

1. A p-4H-SiC or $\mathrm{p}-6 \mathrm{H}-\mathrm{SiC}$ MOS device can be used in accumulation to determine hole effective mass from the hole current versus voltage characteristics [2].

2. An $\mathrm{n}-4 \mathrm{H}-\mathrm{SiC}$ or $\mathrm{n}-6 \mathrm{H}-\mathrm{SiC}$ MOS device can be used in accumulation to determine electron effective mass from electron current versus voltage characteristics [2].

3. An n-Si MOS device can be used in accumulation to determine electron effective mass from the electron current versus voltage characteristics.

4. A p-Si MOS device can be used in accumulation to determine hole effective mass from the hole current versus voltage characteristics. Here, all the metals available as gate contact have a lower electron barrier to oxide conduction band as compared to the hole barrier of $4.6 \mathrm{eV}$ at the $\mathrm{Si}$ anode. Therefore the p-MOS device in accumulation will not have the hole tunnelling current as the dominant current. The choice of gate material should be $\mathrm{p}+$ poly silicon carbide. This will ensure a $6 \mathrm{eV}$ valence electron barrier to the oxide conduction band and the dominant FN hole tunnelling from the Si anode having a $4.6 \mathrm{eV}$ hole barrier will occur at an onset field of about $14 \mathrm{MV} / \mathrm{cm}$.

5. An n-channel $\mathrm{n}+$ polysilicon gated Si MOSFET having a thin oxide of 5 to $10 \mathrm{~nm}$ can be used to determine electron effective mass from the gate tunnelling current and the hole effective mass from the substrate current. This is demonstrated in the present study.

6. A p-4H-SiC based MOS device in inversion can be used to find electron effective mass from the dominant electron current versus voltage characteristics. This method is complex because strong inversion in $\mathrm{SiC}$ does not take place without the application of temperature or shinning UV light. This is due to the fact that the intrinsic carrier concentration in $\mathrm{SiC}$ is low because of its wide band gap.

\section{Conclusion:}

The parabolic electron and hole effective masses in the thermal oxide are determined to be $0.42 \mathrm{~m}$ and $0.58 \mathrm{~m}$ for a free Fermi gas model of carriers at the emitting electrode utilizing a Si n-channel MOSFET device in inversion that allows carrier separation. These carrier masses are related to the band offsets in $\mathrm{SiO}_{2} / \mathrm{Si}<100>$ MOS devices accurately though the MOSFET model and is reported for the first time. For quantum confinement of carriers at the emitting electrode when biased, the electron and hole effective masses in the oxide corrects to $0.51 \mathrm{~m}$ and $0.65 \mathrm{~m}$, respectively, keeping in view that quantum correction is valid for extremely thin channels in MOSFETs. The effective masses are predicted to be the same for all thicknesses of the oxide. The device configurations discussed can be utilized with other insulating materials as well for the determination of carrier masses. Also, the 1/E model of the anode hole injection over the hole barrier of $4.6 \mathrm{eV}$ completely explains the oxide breakdown in thin oxides of 5 to $10 \mathrm{~nm}$ having a slope constant of $516 \mathrm{MV} / \mathrm{cm}$.

\section{References}

[1]. H.S.Chang, H.D.Yang, H.Hwang, H.M.Cho, H.J.Lee andD.W.Moon, "Measurement of the physical and electrical thickness of ultrathin gate oxides", J.Vac.Sci.Technol., vol. 20,pp. 1836-1842, Sep. 2002.

[2]. R.K. Chanana, "Determination of hole effective mass in $\mathrm{SiO}_{2}$ and $\mathrm{SiC}$ conduction band offset using Fowler-Nordheim tunneling characteristics across metal-oxide-semiconductor structures after applying oxide field corrections", J. of Applied Physics, vol. 109, pp. 104508-1 to -6, May 2011.

[3]. M. Lenzlinger and E.H. Snow, "Fowler-Nordheim tunneling into thermally grown $\mathrm{SiO}_{2}$ ", J. of Applied Physics, vol. 40, pp. 278283, Jan. 1969

[4]. R.B. Laughlin, "Optical absorption edge of $\mathrm{SiO}_{2}$ ", Physical Review B, vol. 22, pp. 3021-3029, Sep. 1980.

[5]. V.V. Afanasev, "Valence band offset and hole injection at the $4 \mathrm{H}-, 6 \mathrm{H}-\mathrm{SiC} / \mathrm{SiO}_{2}$ interfaces", Applied Physics Letters, vol. 77, pp. 2024-2026, Sep. 2000. 
Determination of electron and hole effective masses in thermal oxide utilizing an n-channel...

[6]. Y.T. Hou, M.F. Li, Y. Jin, and W.H. Lai, "Direct tunneling hole currents through ultrathin gate oxides in metal-oxidesemiconductor devices", J. of Applied Physics, vol. 91, pp. 258-264, Jan. 2002.

[7]. Z.A. Weinberg, "On tunneling in metal-oxide-silicon structures", J. of Applied Physics, vol. 53, pp. 5052-5056, Jul. 1982.

[8]. S.Rodriguez, J.A.Lopez-Villanueva, I.Melchor andJ.E. Carceller, "Hole confinement and energy subbands in silicon inversion layer using the effective mass theory", J.Appl. Phys., vol. 86, pp. 438-444, Jul. 1999.

[9]. B.Eitan and A.Kolodny, "Two components of tunneling current in metal-oxide-semiconductor structures", Appl. Phys. Lett., vol. 43, pp. 106-108, Jul. 1983 .

[10]. R.K.Chanana, K.McDonald, M.Di Ventra, S.T.Pantelides,L.C.Feldman,G.Y.Chung, C.C.Tin, J.R.Williams, R.A.Weller, "FowlerNordheim hole tunneling in p-SiC/SiO 2 structures", Appl. Phys. Lett., vol. 77, pp. 2560-2562, Oct. 2000.

[11]. R.Waters and B.Van Zeghbroeck, "Fowler-Nordheim tunneling of holes through thermally grown $\mathrm{SiO}_{2}$ on p $\mathrm{p}^{+} 6 \mathrm{H}-\mathrm{SiC}$ ", Appl. Phys. Lett., vol. 73, pp. 3692-3694, Dec. 1998.

[12]. I.D.Parker, "Carrier tunneling and device characteristics in polymer light-emitting diodes", J.Appl. Phys., vol. 75, pp. 1656-1666, Feb. 1994.

[13]. Ben G. Streetman, Sanjay Banerjee, "Field-Effect transistors", in Solid State ElectronicDevices, $5^{\text {th }}$ edition, New Delhi, India, Prentice-Hall, 2001, pp. 267-277.

[14]. M.Depas, B.Vermeire, P.W.Mertens, R.L. Van Meirhaeghe,andM.M. Heyns, "Determination of tunneling parameters in ultra-thin oxide layer poly-Si/SiO $2 / \mathrm{Si}$ structures", Solid-State Electronics, vol. 38, pp. 1465-1471, Aug. 1995.

[15]. D.J. DiMaria, E. Cartier, and D.A. Buchanan, “Anode hole injection and trapping in silicon doxide", J. Appl. Phys., vol. 80, pp. 304-317, Jul. 1996.

[16]. S.M. Sze, 'Unipolar Devices', in Semiconductor Devices, Physics and Technology, New York, John Wiley and Sons,1985, pp. 186-221.

[17]. M.L.Green, E.P.Gusev, R.Degraeve, E.L.Garfunkel, "Ultrathin $(<4 \mathrm{~nm}) \mathrm{SiO}_{2}$ and $\mathrm{Si}-\mathrm{O}-\mathrm{N}$ gate dielectric layers for silicon microelectronics: Understanding the processing, structure, and physical and electrical limits", J.Appl.Phys., vol. 90, pp. 2057-2121, Sep. 2001.

[18]. A. Schenk and G. Heiser, "Modeling and simulation of tunnelling through ultra-thin gate dielectrics", J. Appl. Phys.,vol. 81, pp.7900-7908, Jun. 1997.

[19]. F.A.Noor, M.Abdullah, Sukirno and Khairurrijal, "Analysis of electron direct tunneling current through very-thin gate oxide in MOS capacitors with the parallel-perpendicular kinetic energy components and anisotropic masses", BrazilianJournal of Physics, vol. 40, pp. 404-407, Dec. 2010.

[20]. M.Rasras, I. DeWolf, G. Groeseneken, B. Kaczer, R. Degraeve, and H.E. Maes, "Photo-carrier generation as the origin of FowlerNordheim-induced substrate hole current in thin oxides", IEEE, Trans. On Electron Devices, vol. ED-48, pp. 231-238, Feb. 2001.

[21]. A. Dalla Serra, P. Palestri, L. Selmi, "Can photoemission/absorption processes explain the substrate current of tunneling MOS capacitors?”, Solid-State Electronics, vol. 46, pp. 1069-1073, Jul. 2002.

[22]. K. Kobayashi, A. Teramoto, M. Hirayama, and Y. Fujita, "Model for the substrate hole current based on thermionic hole emission from the anode during Fowler-Nordheim electron tunnelling in n-channel metal-oxide-semiconductor field-effect transistors", J. Appl. Phys., vol. 77, pp.3277-3282, Apr. 1995.

[23]. E.Aderstedt, Per Lundgren, "High-frequency operation potential of the tunnel emitter transistor, Solid-State Electronics, vol. 46, pp.1033-1037, Jul., 2002.

[24]. V.A. Gritsenko, R.M. Ivanov, Yu. N. Morokov, "Electronic structure of amorphous $\mathrm{SiO}_{2}$ : Experiment and numerical simulation", Journal of Theoretical and Experimental Physics, vol. 81, pp. 1208-1216, Dec. 1995.

[25]. J.W. Keister, J.E. Rowe, J.J. Kolodziej, H. Niimi, T.E. Madey, G. Lucovsky, "Band offsets for ultrathin $\mathrm{SiO}_{2}$ and $\mathrm{Si}_{3} \mathrm{~N}_{4}$ films on $\mathrm{Si}(111)$ and $\mathrm{Si}(100)$ from photoemission spectroscopy”, J. Vac. Sci. Technol. B. 17, pp.1831-1835, Jul/Aug 1999.

[26]. E. Bersch, S. Rangan, R.A. Bartynski, E. Garfunkel, E.Vescovo, "Band offsets of ultrathin high-K oxide films with Si", Physical Review B, vol. 78, pp.085114-1 to 10, 2008.

[27]. Yee-Chia Yeo, Qiang Lu, and Chenming Hu, "MOSFET gate oxide reliability: Anode hole injection model and its applications", International Journal Of High Speed Electronics and Systems, vol. 11, no.3, pp. 849-886, 2001

[28]. G.D. Wilk, R.M. Wallace, J.M. Anthony, "High-K gate dielectrics: Current status and materials properties considerations", J. Appl. Phys, vol.89, pp.5243-5275, May 2001.

[29]. J. Robertson, "High dielectric constant oxides", Eur. Phys. J. Appl. Phys., vol. 28, pp.265-291, Dec. 2004. 